\title{
PLC-gamma-1 phosphorylation status is prognostic of metastatic risk in patients with early-stage Luminal-A and $-B$ breast cancer subtypes
}

Rossano Lattanzio ${ }^{1,2^{*}+}$ D, Manuela lezzi ${ }^{2,3+}$, Gianluca Sala ${ }^{1,2}$, Nicola Tinari ${ }^{1,2}$, Marco Falasca ${ }^{4}$, Saverio Alberti ${ }^{5}$, Simonetta Buglioni ${ }^{6}$, Marcella Mottolese ${ }^{6}$, Letizia Perracchio ${ }^{6}$, Pier Giorgio Natali ${ }^{2}$ and Mauro Piantelli ${ }^{2}$

\begin{abstract}
Background: Phospholipase CY1 (PLCY1) is highly expressed in human tumours. Our previous studies reported that both stable and inducible PLCY1 down-regulation can inhibit formation of breast-cancer-derived experimental lung metastasis. Further, high expression of PLCY1 and its constitutively activated forms (i.e., PLCY1-pY1253, PLCY1-pY783) is associated with worse clinical outcome in terms of incidence of distant metastases, but not of local relapse in T1T2, NO breast cancer patients.
\end{abstract}

Methods: In the present retrospective study, we analysed the prognostic role of PLCY1 in early breast cancer patients stratified according to the St. Gallen criteria and to their menopausal status. PLCY1-pY1253 and PLCY1-pY783 protein expression levels were determined by immunohistochemistry on tissue microarrays, and were correlated with patients' clinical data, using univariate and multivariate statistical analyses.

Results: In our series, the prognostic value of PLCY1 overexpression was restricted to Luminal type tumours. From multivariate analyses, PY1253-PLCY1 ${ }^{\text {High }}$ was an independent prognostic factor only in postmenopausal patients with Luminal-B tumours (hazard ratio [HR], 2.4; 95\% confidence interval [Cl], 1.1-5.3; $P=0.034$ ). Conversely, PLCY1-pY783 High was a remarkably strong risk factor $(\mathrm{HR}, 20.1 ; 95 \% \mathrm{Cl}, 2.2-178.4 ; P=0.003)$ for pre/perimenopausal patients with Luminal-A tumours.

Conclusions: $\mathrm{PLC} Y 1$ overexpression is a strong predictive surrogate marker of development of metastases in early Luminal-A and -B breast cancer patients, being able to discriminate patients with high and low risk of metastases. Therefore, targeting the PLCY1 pathway can be considered of potential benefit for prevention of metastatic disease.

Keywords: Breast cancer, Phospholipase CY1, Prognosis, Luminal subtypes, Menopausal status

\section{Background}

Breast cancer incidence accounts for approximately $30 \%$ of all new cancer cases and $14 \%$ of all cancer-related deaths among women worldwide [1]. Implementation of screening programmes can detect early stage, nodenegative (N0) tumours at low-risk of relapse, while

\footnotetext{
* Correspondence: rossano.lattanzio@unich.it

${ }^{\dagger}$ Rossano Lattanzio and Manuela lezzi contributed equally to this work.

'Department of Medical, Oral and Biotechnological Sciences, 'G. d'Annunzio'

University of Chieti-Pescara, Chieti, Italy

${ }^{2}$ Center for Advanced Studies and Technology (CAST), 'G. d'Annunzio'

University of Chieti-Pescara, Via Luigi Polacchi 11, 66100 Chieti, Italy

Full list of author information is available at the end of the article
}

advances in adjuvant treatments can promote decreased mortality from breast cancer [2, 3]. Nevertheless, almost $80 \%$ of women diagnosed with early stage disease are currently treated by breast-conserving surgery alone or combined with adjuvant therapy [4]. In these patients without nodal metastasis (N0), identification of high-risk patients for breast cancer relapse through use of established prognostic factors (i.e., age, tumour size and grade, hormone receptor status) cannot predict prognosis accurately. Moreover, nearly $90 \%$ of patients with cancer limited to the breast receive adjuvant treatments, although only $30 \%$ of these patients will ultimately

(c) The Author(s). 2019 Open Access This article is distributed under the terms of the Creative Commons Attribution 4.0 International License (http://creativecommons.org/licenses/by/4.0/), which permits unrestricted use, distribution, and 
relapse [5-7]. Currently, adjuvant chemotherapy regimens are standard of care for treatment of early-stage disease that is oestrogen receptor (ER)-negative or human epidermal growth factor receptor (HER)2-positive. However, selecting chemotherapy for patients with ERpositive, HER2-negative disease is a more challenging task, due to the different risk profiles for disease relapse associated with this tumour subtype. Indeed, among patients with luminal tumours, there will be women at low risk of recurrence who will derive little benefit from chemotherapy combined with hormone therapy, and women at high-risk of recurrence where chemotherapy would be helpful. As far as the economic impacts on health care systems, is should be noted that adjuvant treatments are not devoid of toxicity. On this basis, above the search for new discriminatory biomarkers capable of selecting patients at risk of early relapse remains mandatory.

Among the lipid signalling metabolites in cells, the phosphoinositides are the most widely studied lipids due to their involvement in several cell signalling pathways. In particular, phosphoinositide-specific phospholipase $\mathrm{C}$ gamma 1 ( $P L C \gamma 1$ ) signalling is necessary for many physiological cellular processes (e.g., cell proliferation and differentiation) [8]. PLC $\gamma 1$ is highly expressed in various tumours, including breast cancers [9-11]. We previously observed that $\mathrm{PLC} \gamma 1$ down-regulation strongly reduced formation of MDA-MB-231-derived lung metastases in nude mice [12]. Furthemore, lung metastasis formation from prostate cancer cells was significantly reduced by a dominant-negative fragment of PLC $\gamma 1$ [13].

In human, using tumour cases as training and validation sets, we have shown that overexpression of activated PLC $\gamma 1$ is a risk factor for distant relapse in T1-T2, No breast cancer patients undergoing adjuvant chemotherapy [14]. Therefore, in the present study, we investigated the prognostic role of PLC $\gamma 1$ in these early breast cancer patients stratified according to the St. Gallen criteria and to their menopausal status.

\section{Methods}

\section{Patients}

We retrospectively reviewed the medical records of 979 consecutive patients (year range, 1995-2003) diagnosed with primary unilateral breast carcinoma at the "Regina Elena" National Cancer Institute, Rome, Italy. From the original series, only N0 patients with T1/T2 tumours were included in the present study $(n=414)$. The patients' and tumour characteristics are given in Table 1.

This study was reviewed and approved by the Ethics Committee of the Regina Elena National Cancer Institute. All of the patients were treated with quadrantectomy and received radiation therapy $(n=414)$, while 172 received chemotherapy without or with hormonal therapy, and 160 underwent only hormonal therapy. Patients with HER2-positive tumours did not receive trastuzumab, because this immune treatment was not available during the study period. The median follow-up was 79 months (range, 2-298 months). Follow-up data were collected from institutional records or from the referring physicians. During follow-up, 50 patients (12.1\%) experienced local relapse. Distant relapse was seen in 65 $(15.7 \%)$ of the patients.

\section{Immunohistochemistry}

The 414 breast cancer cases were distributed in 21 tissue microarrays (TMA) blocks assembled in duplicate. Briefly, TMAs were constructed by punching $2-\mathrm{mm}$ diameter cores of histologically proven invasive breast carcinoma areas, as previously described [14]. The tissue microarray sections were incubated with the mouse antiPLC 1 monoclonal antibody (sc-7290), and with the rabbit anti-PLC 1 1-pY1253 (sc-22141-R) and antiPLC $\gamma 1-p Y 783$ (sc-12943-R) polyclonal antibodies, with all from Santa Cruz Biotechnology (Santa Cruz, CA, USA). Although these antibodies were validated by Santa Cruz Biotechnology, their specificities were further validated using appropriate silenced breast cancer cell lines (Additional file 2: Figure S1). The anti-mouse and anti-rabbit EnVision kits (Agilent, Santa Clara, CA, USA) were used for signal amplification, as appropriate. For the control tissues, the primary antibody was excluded or substituted with non-immune serum or isotype-matched immunoglobulins. The immunohistochemical analysis was carried out by two pathologists (R.L., M.P.) by agreement, with both blinded to the clinicopathological information. The immunohistochemical results for the ER, progesterone receptor (PgR), Ki67 and HER2 status were obtained from the patients' hospital records.

\section{Statistical methods}

The St. Gallen criteria [15] were used to dichotomise the tumour size and tumour grade, as well as the ER, PgR and Ki-67 protein expression. We also examined the distribution of the expression of PLC 1 and its phosphorylated forms in four breast cancer molecular subtypes: Luminal-A $(n=156)$, Luminal-B $(n=176)$, HER2 $(n=27)$ and Triple Negative $(n=55)$. The expression of the PLC 1 , PLC $\gamma 1-\mathrm{pY} 1253$ and PLC $11-$ pY783 proteins were reported as percent of positive cells, and dichotomised (high vs. low) according to the cut-off values corresponding to the 50th (i.e., $75 \%$ of positive cells for PLC 1 and $61 \%$ of positive cells for PLC $\gamma 1-$ pY1253) and 75th (i.e., 59\% of positive cells for PLC 1 1-pY783) percentiles, as previously defined [14]. In all immunohistochemical evaluations, interobserver agreement was scored as near- 
Table 1 Patients and tumor characteristics $(n=414)$

\begin{tabular}{|c|c|}
\hline Variable & Value (\%) \\
\hline \multicolumn{2}{|l|}{ Age at diagnosis (yr) } \\
\hline Median & 59.7 \\
\hline$<50$ & $103(24.9)$ \\
\hline $50-65$ & $173(41.8)$ \\
\hline$>65$ & 138 (33.3) \\
\hline \multicolumn{2}{|l|}{ Menopausal status } \\
\hline Pre/perimenopausal & 109 (26.3) \\
\hline Postmenopausal & $305(73.7)$ \\
\hline \multicolumn{2}{|l|}{ Molecular subtypes } \\
\hline Luminal A & $156(37.7)$ \\
\hline Luminal B & $176(42.5)$ \\
\hline HER2 & $27(6.5)$ \\
\hline Triple negative & $55(13.3)$ \\
\hline \multicolumn{2}{|l|}{ Tumour size } \\
\hline$\leq 2 \mathrm{~cm}$ & $272(65.7)$ \\
\hline$>2 \mathrm{~cm}$ & $142(34.3)$ \\
\hline \multicolumn{2}{|l|}{ Histotypes } \\
\hline Ductal carcinoma & $330(79.7)$ \\
\hline Lobular carcinoma & $54(13.0)$ \\
\hline Other & $30(7.3)$ \\
\hline \multicolumn{2}{|l|}{ Tumour grade } \\
\hline 1 & $64(15.5)$ \\
\hline $2-3$ & $350(84.5)$ \\
\hline \multicolumn{2}{|l|}{ ER } \\
\hline Negative & $89(21.5)$ \\
\hline Positive & $325(78.5)$ \\
\hline \multicolumn{2}{|l|}{$\mathrm{PgR}$} \\
\hline Negative & $188(45.4)$ \\
\hline Positive & $226(54.6)$ \\
\hline \multicolumn{2}{|l|}{ Ki-67 } \\
\hline Low & $287(69.3)$ \\
\hline High & $127(30.7)$ \\
\hline \multicolumn{2}{|l|}{ HER2 } \\
\hline Negative & $348(84.1)$ \\
\hline Positive & $66(15.9)$ \\
\hline \multicolumn{2}{|l|}{ PLCY1 } \\
\hline Low & $225(54.3)$ \\
\hline High & $189(45.7)$ \\
\hline \multicolumn{2}{|l|}{ PLCү1-pY1253 } \\
\hline Low & $241(58.2)$ \\
\hline High & $173(41.8)$ \\
\hline \multicolumn{2}{|l|}{ PLCY1-pY783 } \\
\hline Low & $327(79.0)$ \\
\hline High & $87(21.0)$ \\
\hline
\end{tabular}

Table 1 Patients and tumor characteristics $(n=414)$ (Continued)

\begin{tabular}{ll}
\hline Variable & Value (\%) \\
\hline Patient outcome & \\
Without recurrence & $299(72.2)$ \\
Local recurrence & $50(12.1)$ \\
Distant recurrence & $65(15.7)$ \\
\hline
\end{tabular}

perfect (i.e., PLC 1 1: kappa $=0.854 ; \quad$ PLC $\gamma 1-p Y 1253$ : kappa $=0.861 ;$ PLC 1 1-pY783: kappa $=0.889$ ).

Pearson's $X^{2}$ or Fisher's exact tests were used to asssess the relations between the tumour PLC $\gamma 1$, PLC $\gamma 1$ pY1253 and PLC 1 1-pY783 protein expression and the patient clinicopathological parameters. Disease-free survival (DFS) was defined as the interval from surgery to the first of the following events: tumour relapse at local or distant sites. Local relapse-free survival (LRFS) and distant relapse-free survival (DRFS) were defined as the time from surgery to the occurrence of local and distant relapses, respectively. Kaplan-Meier plots were used for the survival analyses, and log-rank tests were applied to compare the survival curves between the patient groups.

Cox's proportional hazards models were used to evaluate the association of PLC $\gamma 1$, PLC $\gamma 1-p Y 1253$ and PLC $11-p Y 783$ expression with survival time, using covariates. The following covariates were computed in the multivariate models: tumour size, tumour grade, and ER, PgR, Ki-67, HER2, PLC $\gamma 1$, PLC $\gamma 1-p Y 1253$ and PLC $\gamma 1-$ pY783 status. The statistical software SPSS version 15.0 (SPSS, Chicago, IL, USA) was used throughout, and $P<$ 0.05 was considered statistically significant.

\section{Results}

PLCY1, PLCY1-pY1253 and PLCY1-pY783 immunostaining As described previously [14], neoplastic cells presented cytoplasmic immunoreactivity for PLC $\gamma 1$, whereas positivity for PLC 1 1-pY1253 and PLC $\gamma 1-$ pY783 was almost exclusively nuclear (Fig. 1). In all, 189 of 414 (45.7\%) patients showed high tumour PLC $\gamma 1$ expression levels $\left(\mathrm{PLC} \gamma 1^{\text {High }}\right)$. Similarly for tumour expression of PLC $\gamma 1$ pY1253 ${ }^{\mathrm{High}}$ and PLC $11-\mathrm{pY} 783^{\mathrm{High}}$, as $173 / 414$ (41.8\%) and $87 / 414(21.0 \%)$, respectively (Table 1 ).

\section{Survival analysis in early breast cancer patients All cases}

Kaplan-Meier plots showed significant association of high tumour expression of PLC $\gamma 1$, pY1253-PLC $\gamma 1$ and pY783-PLC $\gamma 1$ with low DFS rates $(P=0.005, P=0.019$, $P=0.006$, respectively) (Additional file 2 : Figure S2). In particular, tumours that overexpressed PLC $\gamma 1$ or its activated forms were associated with significantly higher frequency of distant relapse $(P=0.001, P=0.001, P=0.005$, respectively), while no significant correlations with local relapse were observed. 


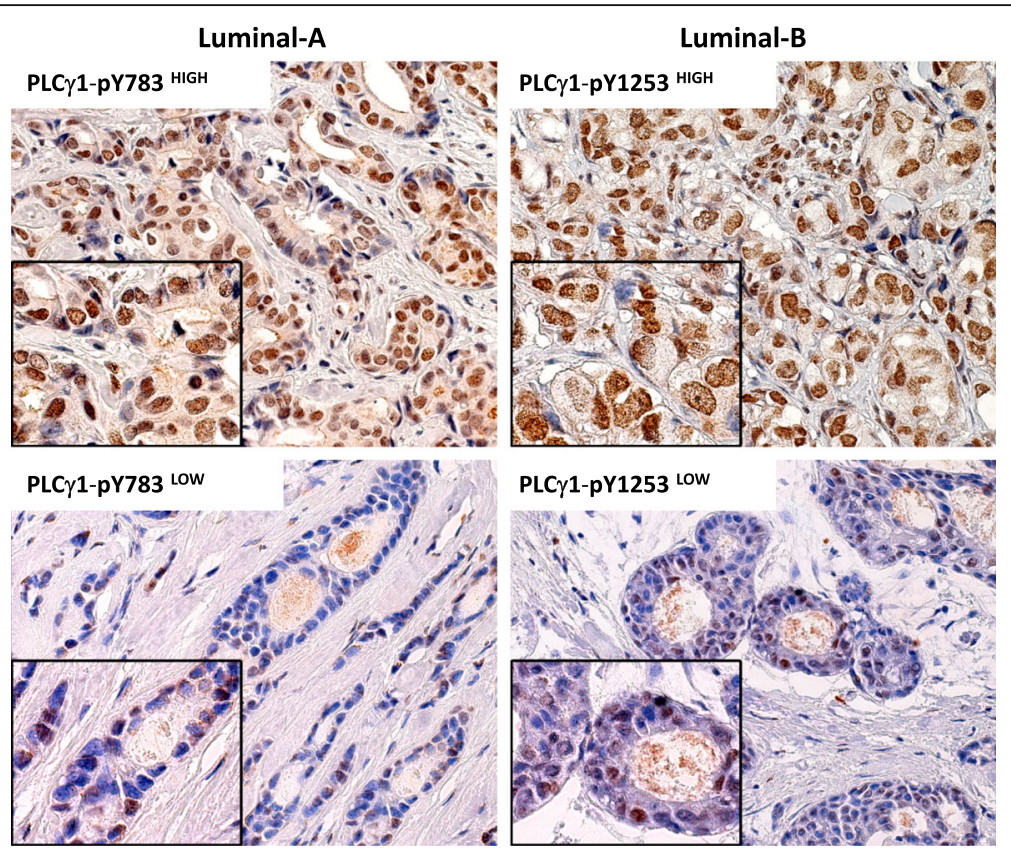

Fig. 1 PLCY1-pY1253 and PLCY1-pY783 immunostaining: examples of high (upper panel) and low (lower panel) expression in Luminal-A and Luminal-B breast cancer subtypes. Expression of PLCY1 phosphorylated forms is confined to tumor cell nuclei

Multivariate analyses of DFS revealed prognostic significance of tumour expression of PLC $\gamma 1$ (HR, 1.5; 95\% CI, $1.0-2.3 ; P=0.029)$, pY1253-PLC 1 (HR, 1.6; 95\% CI, $1.1-2.3 ; P=0.024$ ) and tumour grade (Additional file 1 : Table S1). In addition, higher risk of distant but not of local relapse was seen for PLCY1 $1^{\text {High }}(\mathrm{HR}, 2.1 ; 95 \% \mathrm{CI}$, 1.3-3.6; $P=0.005)$, pY1253-PLC $1^{\text {High }}(\mathrm{HR}, 2.3 ; 95 \% \mathrm{CI}$, $1.4-3.7 ; P=0.001)$ and pY783-PLCY $1^{\text {High }}(\mathrm{HR}, 1.7 ; 95 \%$ CI, $1.0-2.7 ; P=0.049$ ).

\section{Tumour subtypes}

According to the Kaplan-Meier analysis, high expression of PLC $\gamma 1$ or its activated forms was significantly correlated with lower DFS rates in patients with Luminal-A tumours (Fig. 1), but not in patients with Luminal-B tumours (Fig. 3), or HER2 positive (Additional file 2: Figure S3) or Triple Negative tumours (Additional file 2: Figure S4). Significantly higher rates of distant relapse were seen for Luminal-A pY1253-PLCY1 $1^{\text {High }}(P=0.029)$ and pY783PLCY $1^{\text {High }}(P<0.001)$ tumours (Fig. 2). In Luminal-B tumours, those that were pY1253-PLCY $1^{\text {High }}(P=0.016)$, but not those that were pY783-PLC $11^{\text {High }}(P=0.968)$ showed significantly increased risk of distant relapse (Fig. 3). Multivariate analysis showed that high expression of PLCY1pY1253 was an independent prognostic marker for DRFS in Luminal-B tumours (HR, 2.3; 95\% CI, 1.2-4.6; $P=$ 0.017), while only high expression of PLC $11-$ pY783 was correlated with significantly higher risk of distant relapse in patients with Luminal-A tumours (HR, 7.4; 95\% CI, 2.324.3; $P=0.001$ ) (Additional file 1: Table S2).
Kaplan-Meier and multivariate analyses of PLCY1 transcript expression in tumours from the 1,881-sample breast cancer dataset (GOBO; Gene expression-based Outcome for Breast cancer Online; http://co.bmc.lu.se/ gobo) [16], which further confirmed the negative prognostic value of high PLCY1 protein expression in lymphnode-negative Luminal-A tumours $(n=184)(P<0.005$, $P=0.004$, respectively) (Additional file 2: Figure S5). Consistent with this, significantly higher risk of metastatic relapse in patients with Luminal-A, lymph-node negative, PLC $1^{\text {High }}$ tumours $(n=546)$ was also found in the KM-Plotter microarray database (HR, 2.19; 95\% CI, 1.23-3.89; $P=0.0065$ ) (Additional file 2: Figure S6) [17].

\section{Menopausal status}

Using Kaplan-Meier plots, we also examined the distant recurrence rates associated with PLC 1 1-pY1253 and PLC 1 1-pY783 expression in luminal tumours with patients clustered according to menopausal status. We observed that pY1253-PLC $1^{\text {High }}$ expression was significantly associated with lower DRFS rate in postmenopausal patients with Luminal-B tumours $(P=0.028)$, while pY783-PLC $1^{\text {High }}$ expression was significantly correlated with increased risk of distant relapse in those patients with Luminal-A cancers and pre/perimenopausal status $(P<0.001)$ (Fig. 4).

Multivariate analyses confirmed that pY1253-PLC $\gamma 1^{\text {High }}$ was a significant independent prognostic factor for postmenopausal Luminal-B cancers (HR, 2.4: 95\% CI, 1.1-5.3; $P=0.034)$, while over-expression of PLC $11-$ pY783 


\section{Luminal-A}

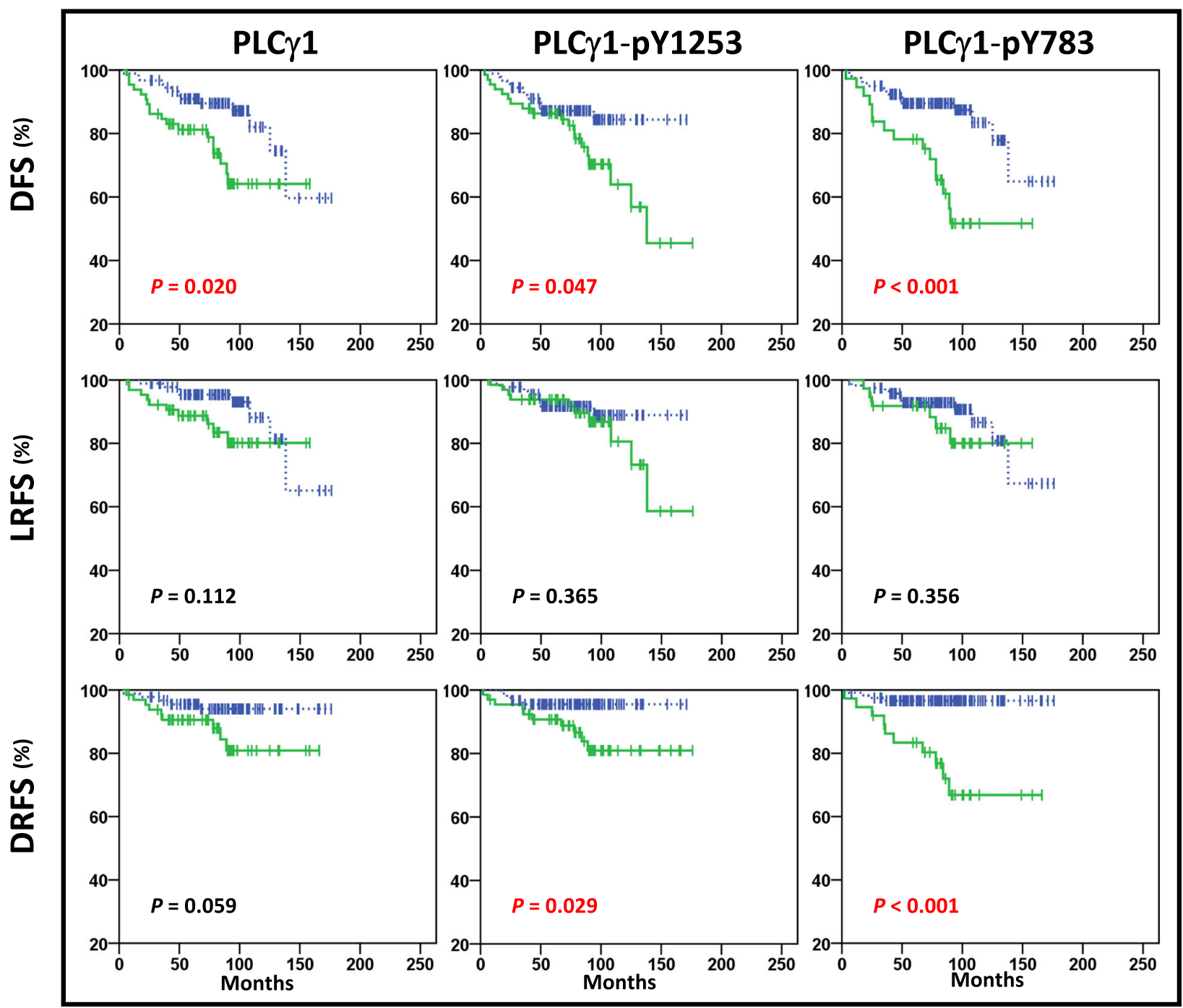

Fig. 2 Kaplan-Meier plots in the Luminal-A subtype. Kaplan-Meier estimates of DFS, LRFS and DRFS in patients with Luminal-A tumours ( $n=156$ ), according to high (solid green lines) and low (dashed blue lines) expression of PLCY1, PLCY1-pY1253 and PLCY1-pY783. In this cohort, the patients showed distant relapse in 6\% (5/91) PLCY ${ }^{\text {Low }}$ and 14\% (9/65) PLCY1 $1^{\text {High }}$, in 4\% (4/90) PLCY1-pY1253 ${ }^{\text {Low }}$ and 15\% (10/66) PLCY1pY1253 ${ }^{\text {High }}$, and in 3\% (4/119) PLCY1-pY783 ${ }^{\text {Low }}$ and 27\% (10/37) PLCY1-pY783 High

represented a significant and strong risk factor for pre/perimenopausal patients with Luminal-A tumours (HR, 20.1: 95\% CI, 2.2-178.4; $P=0.003$ ) (Table 2, Additional file 1: Table S3).

Significant negative prognostic value was seen for pY783-PLC $\gamma 1^{\text {High }}$ for women with Luminal-A tumours who were pre/perimenopausal and were treated with hormonal therapy, as well as those treated with chemotherapy plus hormonal therapy $(P=0.003, P=0.001$, respectively; Fig. 5). The two patients treated with radiotherapy alone were pY783-PLC $\gamma 1^{\text {Low }}$, and they did not show distant metastasis events.

\section{Discussion}

The identification of criteria for accurate prognostication of disease relapse is crucial for the selection of patient candidates for adjuvant therapy. Recognizing patients with high recurrence risk can potentially enhance their treatment outcomes, with the adoption of more aggressive treatments from an earlier stage of the disease, which might ultimately offer better overall survival. Conversely, low-risk patients can undergo less aggressive therapy, and therefore they can enjoy a better quality of life.

PLC 11 is activated through its phosphorylation by tyrosine kinases in the cell. The major tyrosine kinases 


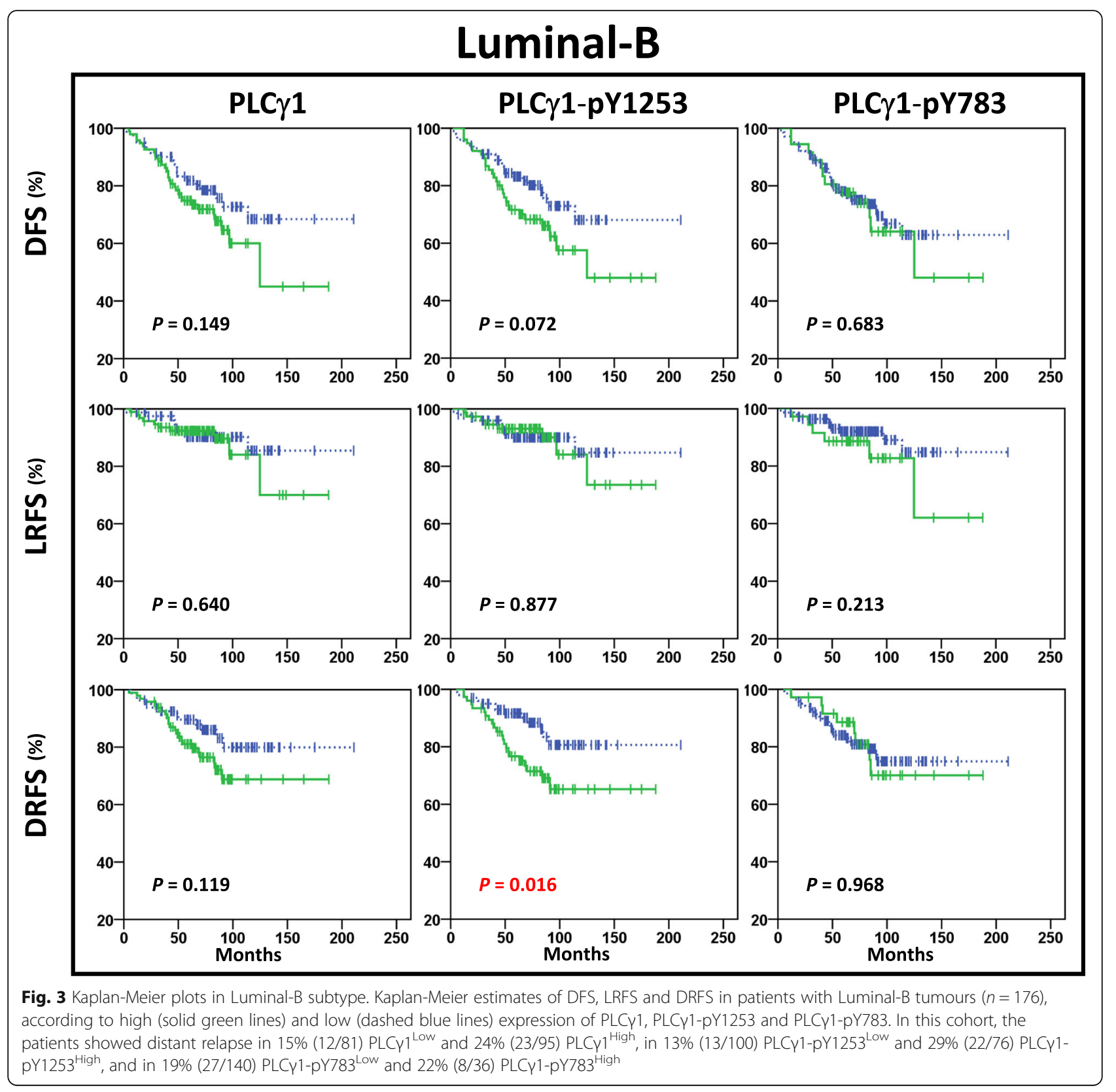

that have been shown to activate PLCY1 in the cell belong to the growth factor receptor superfamily [11], which include the activated receptors for human epidermal growth factor (i.e., HER1/2), fibroblast growth factor, vascular endothelial growth factor, platelet-derived growth factor, hepatocyte growth factor and insulin-like growth factor. Thus, these tyrosine kinase receptors can phosphorylate PLC 1 on its three tyrosine residues: Y771 and Y783 located between the $\mathrm{X}$ and $\mathrm{Y}$ catalytic domains, and Y1253 located near the COOH-terminus domain. Upon phosphorylation, PLC $\gamma 1$ shows increased enzymatic activity, whereby the phosphorylation at Y783 has been described as required for PLC $\gamma 1$ activation in vitro and in vivo [18-20].

Although the phosphoinositide cycle operates classically at the plasma membrane level, a phosphoinositide cycle operates also within the nucleus [21]. We previously observed a selective nuclear positivity for PLC $\gamma 1$ pY1253 and PLC 1 1-pY783 in patients with early breast cancer, indicating that the nuclear signalling of these activated forms of PLC 1 may have a specific tumorigenic role [14]. The nuclear PLC $\gamma 1$ can contribute to mammary carcinogenesis through the modulation of key pathways, including the phosphoinositide 3-kinase 


\section{Distant Relapse Free Survival}

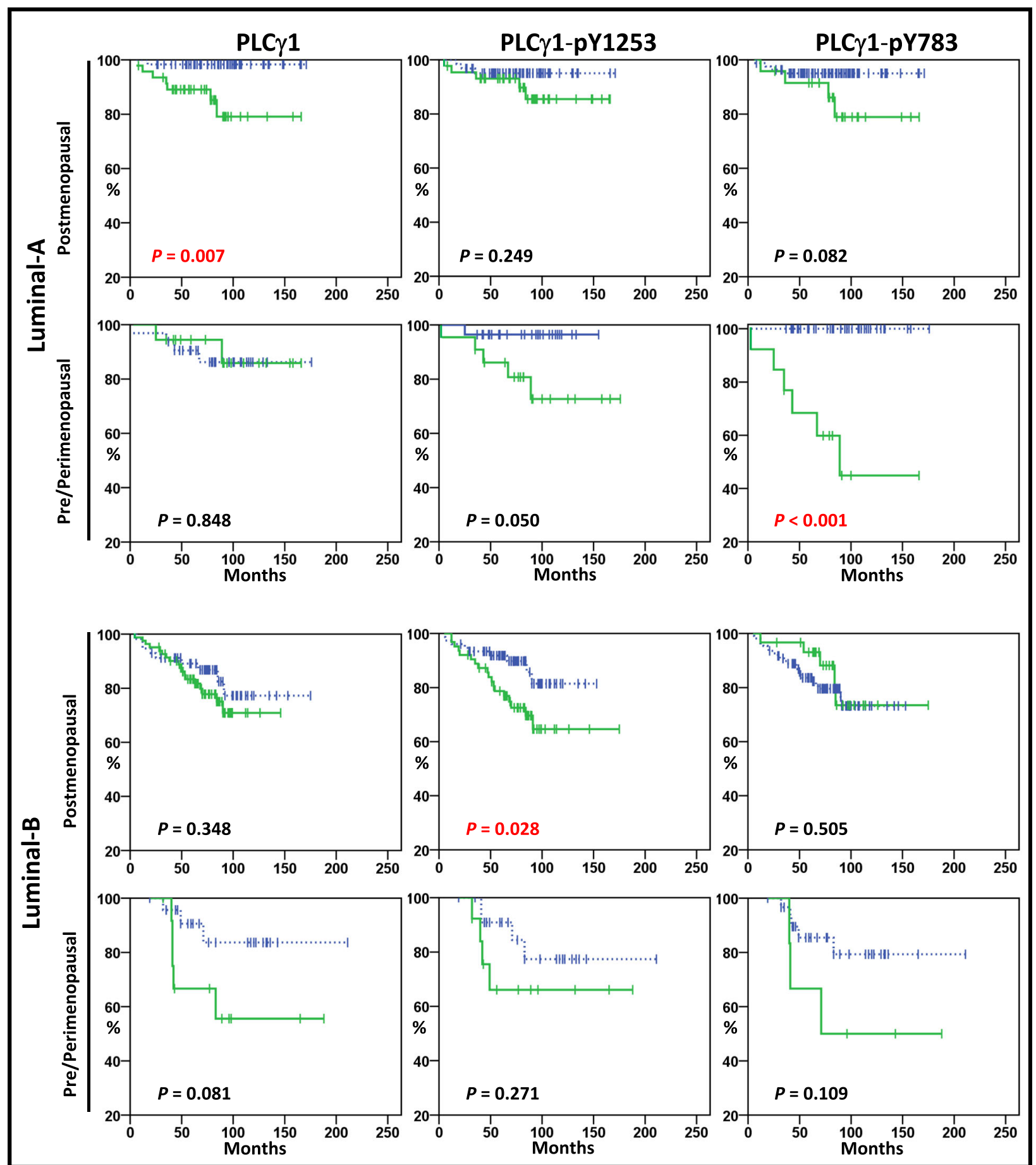

Fig. 4 (See legend on next page.) 
(See figure on previous page.)

Fig. 4 Kaplan-Meier plots in Luminal-A and Luminal-B subtypes. Kaplan-Meier estimates of DRFS in patients with Luminal-A and Luminal-B tumours according to menopausal status. In Luminal-A tumours, the patients with postmenopausal status showed distant relapse in 5\% (3/62) PLCY1-pY1253 ${ }^{\text {Low }}$ and 11\% (5/44) PLCY1-pY1253 ${ }^{\text {High }}$, and in 5\% (4/82) PLCY1-pY783 ${ }^{\text {Low }}$ and 17\% (4/24) PLCY1-pY783 $3^{\text {High }}$, and the patients with premenopausal status showed distant relapse in 4\% (1/28) PLCY1-pY1253 Low and 23\% (5/22) PLCY1-pY1253 High, and in 0\% (0/37) PLCY1-pY783 ${ }^{\text {Low }}$ and 46\% (6/13) PLCY1-pY783 ${ }^{\text {High }}$. In Luminal-B tumours, the patients with postmenopausal status showed distant relapse in 12\% (9/76) PLCY1pY1253 ${ }^{\text {Low }}$ and 29\% (18/63) PLCY1-pY1253 ${ }^{\text {High }}$, and in 20\% (22/109) PLCY1-pY783 ${ }^{\text {Low }}$ and 17\% (5/30) PLCY1-pY783 High, and the patients with premenopausal status showed distant relapse in 17\% (4/24) PLCY1-pY1253 ${ }^{\text {Low }}$ and 31\% (4/13) PLCY1-pY1253 ${ }^{\text {High }}$, and in 16\% (5/31) PLC 1pY783 ${ }^{\text {Low }}$ and 50\% (3/6) PLCY1-pY783 High

(PI3K) nuclear activation [22], and by regulating the expression of cell cycle regulators such as cyclin D1 and cyclin-dependent kinase 4 expression, and the nuclear export of the Cdk inhibitor p27-kip1 [23]. Conversely, the down-regulation of PLC 1 expression in breast cancer cells results in decreased lung metastasis formation in mice [12]. However, the mechanism/s by which PLC $\gamma 1$ favours migration and metastatisation remain unclear. PLC 11 has an essential role in cytoskeletal changes needed for the acquisition of the metastatic phenotype $[11,24]$, and dephosphorylation of PLC $\mathrm{P} 1$ on residue Y783 inhibits PLC $\gamma 1$ activation, thus blocking PLC $\gamma 1$-activated rearrangement of the cytoskeleton, and cell migration [25]. PLC 1 can contribute to metastatisation by direct [26] or indirect $[12,26]$ activation of RAC1, thus inducing migration-supporting cellular structures, such as lamellipodia and filopodia.

In the present study, we have shown that the prognostic role of activated PLCY1 expression is limited to ER-positive, Luminal breast tumours. Indeed, by using validated antibodies for immunohistochemistry on paraffin sections, different PLC $\gamma 1$ phosphorylation sites were associated with different prognosis for the Luminal-A and -B molecular subtypes. Of note, pY1253-PLC $\gamma 1^{\text {High }}$, but not pY783-PLC $\gamma 1^{\text {High }}$, was a significant independent prognostic factor for postmenopausal patients with Luminal B cancers $(H R, 2.4)$. On the other hand, this was reversed in the hormonal pre/perimenopausal setting, where pY783PLC $\gamma 1^{\text {High }}$, but not pY1253-PLC $1^{\text {High }}$, was a particularly strong and significant risk factor for metastatic relapse (HR, 20.1).
Several multigene assays can now be included in clinical practice, such as the Oncotype DX, Prosigna and MammaPrint assays. Compared to the use of standard prognostic criteria, these multigene assays can provide some improvements in the recognition of patients with early stage ER-positive, HER2-negative breast cancer that will be at risk of recurrence. Indeed, at present, randomised controlled trials [27] are ongoing to prospectively validate their clinical usefulness. The future of diagnostic/ prognostic testing in ER-positive breast cancer is likely to rely on devising and reliably deploying assays that can predict the benefits of additional therapies, including newer targeted therapies. As no particular technology holds the key, immunohistochemistry remains a well settled, widely diffuse, and low-cost technique, and so it can have a role in the choice of adequate treatment [28].

Although the relationship between oestrogen stimulation and PLCY expression has been explored in depth, recent data [29] have indicate a role for PLCY1 in the proliferation of ER-positive tumour cells. Cells must increase chaperone levels to fold and sort proteins required for ER $\alpha$-dependent cell proliferation. The unfolded protein response (UPR), which is an endoplasmic reticulum stress sensor, controls protein folding homeostasis. The UPR is overexpressed in several tumours where an early, pathological, activation of UPR occurs before the accumulation of unfolded proteins. In ER $\alpha$ positive breast and ovary cancer cells, 17 $\beta$-oestradiol induces rapid anticipatory activation of the UPR that is strictly PLC $\gamma 1$ dependent. ER-positive breast cancers

Table 2 Risk of distant relapse according to PLCY1-pY1253 and PLCY1-pY783 expression in Luminal-A and Luminal-B tumour subtypes depends on menopausal status. Multivariate analysis

\begin{tabular}{|c|c|c|c|c|c|c|c|}
\hline \multirow[t]{2}{*}{ Menopause status } & \multirow{2}{*}{$\begin{array}{l}\text { Tumour } \\
\text { subtype }\end{array}$} & \multicolumn{3}{|c|}{ PLCY1-pY1253 } & \multicolumn{3}{|c|}{ PLCү1-pY783 } \\
\hline & & $\mathrm{HR}^{\mathrm{a}}$ & $95 \% \mathrm{Cl}^{\mathrm{b}}$ & $P$ & $\mathrm{HR}^{\mathrm{a}}$ & $95 \% \mathrm{Cl}^{\mathrm{b}}$ & $P$ \\
\hline \multirow[t]{2}{*}{ Pre/Perimenopausal } & Luminal A & 7.5 & $0.8-71.8$ & 0.079 & 20.1 & $2.2-178.4$ & 0.003 \\
\hline & Luminal B & 2.5 & $0.6-10.3$ & 0.195 & 2.9 & $0.7-12.2$ & 0.149 \\
\hline \multirow[t]{2}{*}{ Postmenopausal } & Luminal A & 2.2 & $0.5-9.4$ & 0.274 & 2.6 & $0.7-10.7$ & 0.172 \\
\hline & Luminal B & 2.4 & $1.1-5.3$ & 0.034 & 1.2 & $0.5-3.2$ & 0.717 \\
\hline
\end{tabular}

${ }^{\mathrm{a}} H R$ Hazard ratio (high versus low PLCY1-pY expression)

${ }^{\mathrm{b}} \mathrm{Cl}$ Confidence interval; statistically significant $p$-values are formatted in bold 

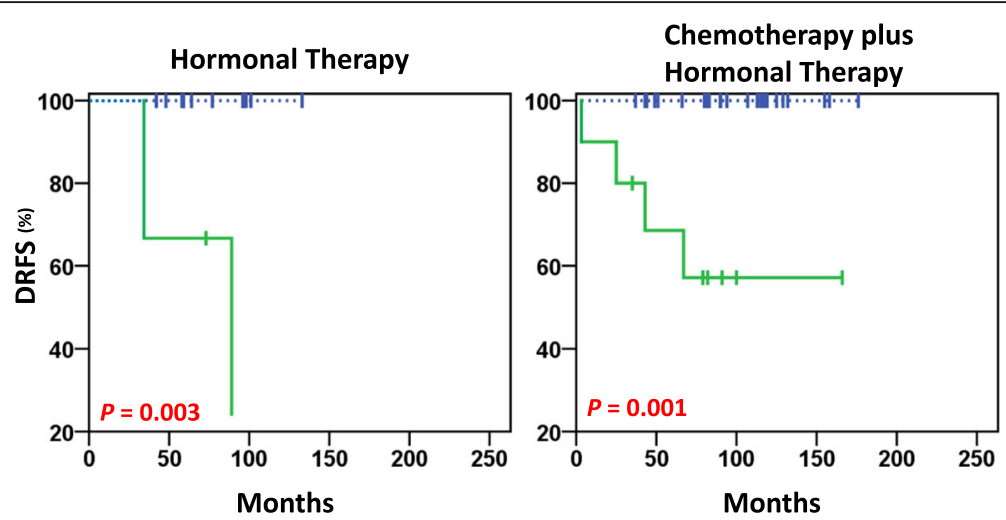

Fig. 5 DRFS estimates in Luminal-A premenopausal patients treated with hormonal therapy alone (left) and chemotherapy plus hormonal therapy (right). In this cohort, a distant relapse occurred in 0\% (0/12) PLCY1-pY783 $3^{\text {Low }}$ and $66 \%(2 / 3)$ PLCY1-pY783 ${ }^{\text {High }}$ of patients treated with hormonal therapy $(n=15)$, and in 0\% (0/23) PLCY1-pY783 $3^{\text {Low }}$ and 40\% (4/10) PLCY1-pY783 $3^{\text {High }}$ of patients treated with chemotherapy plus hormonal therapy $(n=33)$. The solid green line and dashed blue line represent high and low expression of PLCY1-pY783, respectively

demonstrate elevated expression of a UPR gene signature that is also a prognostic marker associated to high risk of relapse and poor survival, and also resistance to tamoxifen therapy. Therefore, this PLC 1 1-dependent anticipatory activation of the UPR defines a new role for oestrogens that can create a supportive environment for cancer cell proliferation and resistance to therapy, and might represent a new target in breast cancer [30]. Considering this aspect, it has also been reported that $\mathrm{PLC} \gamma 1$ activation downstream of FGFR-3 signalling is a critical event in the control of MAPK and PI3K activation, which can induce resistance to tamoxifen treatment [31].

\section{Conclusions}

Although the mechanisms involved remain to be defined, the activation of PLC 1 as assessed by immunohistochemistry is a strong prognostic factor that can discriminate between high-risk and low-risk patients with hormone-receptor-positive early breast cancers. PLC 1 might thus serve as a new target especially for treatment of LuminalA pre/perimenopausal patients with T1-T2, N0 disease.

\section{Additional files}

Additional file 1: Table S1. Multivariate analyses of PLCY1, PLCY1pY1253 and PLCY1-pY783 expression in all cases $(n=414)$. Table S2. Multivariate analyses of PLCY1-pY1253, pY783 and PLCY1 expression in Luminal-A and Luminal-B subtypes. Table S3. PLCY1-pY1253 and PLCY1pY783 expression in Luminal-A (LA) and Luminal-B (LB) subtypes according to menopausal status: multivariate analyses. (DOCX $49 \mathrm{~kb}$ )

Additional file 2: Figure S1. Immunoreactivity for PLCY1, PLCY1-pY1253 and PLCY1-pY783 in wild-type (wt) and down-regulated (si) MDA-MB-231 breast cancer cells. Figure S2. All patients $(n=414)$ : Kaplan-Meier estimates of DFS, LRFS, and DRFS according to high (solid green lines) and low (dashed blue lines) expression of PLCY1, PLCY1-pY1253 and PLCY1-pY783; Figure S3. Patients with HER2 positive breast cancer subtype $(n=27)$ : Kaplan-Meier estimates of DFS, LRFS, and DRFS according to high (solid green lines) and low (dashed blue lines) expression of PLCY1, PLCY1-pY1253 and PLCY1-pY783. Figure S4. Patients with Triple Negative breast cancer subtype $(n=55)$ : Kaplan-Meier estimates of DFS, LRFS, and DRFS according to high (solid green lines) and low (dashed blue lines) expression of PLCY1, PLCY1-pY1253 and PLCY1-pY783. Figure S5. GOBO (Gene expression-based Outcome for Breast cancer Online) database (http://co.bmc.lu.se/gobo): Kaplan-Meier plot of DFS (A) and multivariate (B) analyses of PLCG1 transcript expression in lymph-node-negative HU-Luminal A tumours $(n=184)$. Red and grey lines represent tumours expressing high and low PLCG1 mRNA levels, respectively. Figure S6. KM-Plotter microarray database (http:// kmplot.com/analysis/index.php?p=service\&cancer=breast): Kaplan-Meier plot of distant metastasis-free survival (DMFS) of PLCG1 transcript expression in Luminal-A lymph-node negative breast cancer patients $(n=$ 546). Red and black lines represent tumours expressing high and low PLCG1 mRNA levels, respectively. (PPTX $1483 \mathrm{~kb}$ )

\section{Abbreviations}

Cl: Confidence interval; DFS: Disease-free survival; DRFS: Distant relapse-free survival; ER: Oestrogen receptor; HR: Hazard ratio; LRFS: Local relapse-free survival; PgR: Progesterone receptor; PLCY1: Phospholipase $C Y 1$;

UPR: Unfolded protein response

\section{Acknowledgments}

Not applicable.

\section{Authors' contributions}

$\mathrm{RL}$ and $\mathrm{Ml}$ carried out the experimental design, data analysis and interpretation, and helped with drafting the article; GS, MF and SA helped with data interpretation and drafting of the article; NT carried out the statistical analysis and helped with data interpretation; BS and LP provided samples and information on participants; MM contributed to data interpretation and helped with drafting the article; PGN and MP carried out the study concept and design, study supervision, drafting of the manuscript and its critical revision. All authors have read and approved the final manuscript.

\section{Funding}

This research was supported by Associazione Italiana Ricerca sul Cancro (AIRC) (06/30/C/9) to Pier Giorgio Natali, Mauro Piantelli, Marcella Mottolese, and Gianluca Sala, and by the Mediterranean Taskforce for Cancer Control (www.mtcc-prevention.net). The funding bodies did not have any role in the design of the study, the collection, analysis and interpretation of the data, and the writing of the manuscript. 


\section{Availability of data and materials}

The data that support the findings of this study are not available publicly. However, the data are available from the corresponding author upon reasonable request.

\section{Ethics approval and consent to participate}

This study was approved by the Ethics Committee of "Regina Elena" National Cancer Institute, Rome, Italy. The study was conducted in compliance with the Helsinki Declaration. The use of tissues had been approved by the Ethics Committee. The written consent for the research use of the surgical samples was obtained upon patients' admittance.

\section{Consent for publication}

Not applicable.

\section{Competing interests}

The authors declare that they have no competing interests.

\section{Author details}

'Department of Medical, Oral and Biotechnological Sciences, 'G. d'Annunzio' University of Chieti-Pescara, Chieti, Italy. ${ }^{2}$ Center for Advanced Studies and Technology (CAST), 'G. d'Annunzio' University of Chieti-Pescara, Via Luigi Polacchi 11, 66100 Chieti, Italy. ${ }^{3}$ Department of Medicine and Aging Sciences, 'G. d'Annunzio' University of Chieti-Pescara, Chieti, Italy. ${ }^{4}$ Metabolic Signalling Group, School of Pharmacy and Biomedical Sciences, Curtin Health Innovation Research Institute, Curtin University, Perth, Australia. ${ }^{5}$ Department of Biotechnology BIOMORF, University of Messina, Via Consolare Valeria 1, 98125 Messina, Italy. 'Department of Pathology, 'Regina Elena' National Cancer Institute, Via E. Chianesi, 53, 00144 Rome, Italy.

Received: 29 December 2018 Accepted: 17 July 2019

Published online: 30 July 2019

\section{References}

1. Siegel R, Naishadham D, Jemal A. Cancer statistics, 2013. CA Cancer J Clin. 2013;63:11-30.

2. Botha JL, Bray F, Sankila R, Parkin DM. Breast cancer incidence and mortality trends in 16 European countries. Eur J Cancer. 2003;39:1718-29.

3. Peto R, Boreham J, Clarke M, Davies C, Beral V. UK and USA breast cancer deaths down 25\% in year 2000 at ages 20-69 years. Lancet. 2000;355:1822.

4. Early Breast Cancer Trialists' Collaborative Group (EBCTCG). Effects of chemotherapy and hormonal therapy for early breast cancer on recurrence and 15-year survival: an overview of the randomised trials. Lancet. 2005;365: 1687-717.

5. Cianfrocca M, Goldstein LJ. Prognostic and predictive factors in early-stage breast cancer. Oncologist. 2004;9:606-16.

6. Eifel P, Axelson JA, Costa J, Crowley J, Curran WJ Jr, Deshler A, Fulton S, Hendricks CB, Kemeny M, Kornblith AB, et al. National Institutes of Health consensus development conference statement: adjuvant therapy for breast cancer, November 1-3, 2000. J Natl Cancer Inst. 2001;93:979-89.

7. Goldhirsch A, Glick JH, Gelber RD, Coates AS, Thurlimann B, Senn HJ. Meeting highlights: international expert consensus on the primary therapy of early breast cancer 2005. Ann Oncol. 2005;16:1569-83.

8. Yang YR, Choi JH, Chang JS, Kwon HM, Jang HJ, Ryu SH, Suh PG. Diverse cellular and physiological roles of phospholipase C-gamma1. Adv Biol Regul. 2012;52:138-51.

9. Eccles SA. Targeting key steps in metastatic tumour progression. Curr Opin Genet Dev. 2005;15:77-86.

10. Emmanouilidi A, Lattanzio R, Sala G, Piantelli M, Falasca M. The role of phospholipase Cgamma1 in breast cancer and its clinical significance. Future Oncol. 2017. https://doi.org/10.2217/fon-2017-0125.

11. Wells A, Grandis JR. Phospholipase C-gamma1 in tumour progression. Clin Exp Metastasis. 2003;20:285-90.

12. Sala G, Dituri F, Raimondi C, Previdi S, Maffucci T, Mazzoletti M, Rossi C, lezzi M, Lattanzio R, Piantelli M, et al. Phospholipase Cgamma1 is required for metastasis development and progression. Cancer Res. 2008;68:10187-96.

13. Shepard CR, Kassis J, Whaley DL, Kim HG, Wells A. PLC gamma contributes to metastasis of in situ-occurring mammary and prostate tumours. Oncogene. 2007;26:3020-6.

14. Lattanzio R, Marchisio M, La Sorda R, Tinari N, Falasca M, Alberti S, Miscia S, Ercolani C, Di Benedetto A, Perracchio L, et al. Overexpression of activated phospholipase Cgamma1 is a risk factor for distant metastases in T1-T2, N0 breast cancer patients undergoing adjuvant chemotherapy. Int J Cancer. 2013;132:1022-31.

15. Goldhirsch A, Winer EP, Coates AS, Gelber RD, Piccart-Gebhart M, Thurlimann B, Senn HJ. Personalizing the treatment of women with early breast cancer: highlights of the St Gallen international expert consensus on the primary therapy of early breast cancer 2013. Ann Oncol. 2013;24:2206-23.

16. Ringner M, Fredlund E, Hakkinen J, Borg A, Staaf J. GOBO: gene expressionbased outcome for breast cancer online. PLoS One. 2011;6:e17911.

17. Gyorffy B, Lanczky A, Eklund AC, Denkert C, Budczies J, Li Q, Szallasi Z. An online survival analysis tool to rapidly assess the effect of 22,277 genes on breast cancer prognosis using microarray data of 1,809 patients. Breast Cancer Res Treat. 2010;123:725-31.

18. Kim HK, Kim JW, Zilberstein A, Margolis B, Kim JG, Schlessinger J, Rhee SG. PDGF stimulation of inositol phospholipid hydrolysis requires PLC-gamma 1 phosphorylation on tyrosine residues 783 and 1254. Cell. 1991;65:435-41.

19. Nishibe S, Wahl MI, Hernandez-Sotomayor SM, Tonks NK, Rhee SG, Carpenter G. Increase of the catalytic activity of phospholipase C-gamma 1 by tyrosine phosphorylation. Science. 1990;250:1253-6.

20. Poulin B, Sekiya F, Rhee SG. Intramolecular interaction between phosphorylated tyrosine-783 and the C-terminal Src homology 2 domain activates phospholipase C-gamma1. Proc Natl Acad Sci U S A. 2005;102:4276-81.

21. Keune W, Bultsma Y, Sommer L, Jones D, Divecha N. Phosphoinositide signalling in the nucleus. Adv Enzym Regul. 2011;51:91-9.

22. Ye K, Aghdasi B, Luo H, Moriarity J, Wu F, Hong J, Hurt K, Bae S, Suh P, Snyder S. Phospholipase Cg1 is a physiological guanine nucleotide exchange factor for the nuclear GTPase PIKE. Nature. 2002;415:541-4.

23. Jang H, Suh P, Lee $Y$, Shin K, Cocco L, Chae Y. PLCY1: potential arbitrator of cancer progression. Adv Biol Regul. 2018;67:179-89.

24. Yamaguchi $\mathrm{H}$, Condeelis J. Regulation of the actin cytoskeleton in cancer cell migration and invasion. Biochim Biophys Acta. 2007;1773:642-52.

25. Phillips-Mason PJ, Kaur H, Burden-Gulley SM, Craig SE, Brady-Kalnay SM. Identification of phospholipase $C$ gamma1 as a protein tyrosine phosphatase mu substrate that regulates cell migration. J Cell Biochem. 2011;112:39-48.

26. Li S, Wang Q, Wang Y, Chen X, Wang Z. PLC-gamma1 and Rac1 coregulate EGF-induced cytoskeleton remodeling and cell migration. Molec Endocrinol. 2009;23:901-13.

27. Kwa M, Makris A, Esteva FJ. Clinical utility of gene-expression signatures in early stage breast cancer. Nat Rev Clin Oncol. 2017;14:595-610.

28. Bhargava R, Brufsky AM, Davidson NE. Prognostic/predictive immunohistochemistry assays for estrogen receptor-positive breast cancer: back to the future? J Clin Oncol. 2012;30:4451-3.

29. Andruska N, Zheng X, Yang X, Helferich WG, Shapiro DJ. Anticipatory estrogen activation of the unfolded protein response is linked to cell proliferation and poor survival in estrogen receptor alpha-positive breast cancer. Oncogene. 2015;34:3760-9.

30. Andruska ND, Zheng X, Yang X, Mao C, Cherian MM, Mahapatra L, Helferich WG, Shapiro DJ. Estrogen receptor alpha inhibitor activates the unfolded protein response, blocks protein synthesis, and induces tumor regression. Proc Natl Acad Sci U S A. 2015;112:4737-42.

31. Tomlinson DC, Knowles MA, Speirs V. Mechanisms of FGFR3 actions in endocrine-resistant breast cancer. Int J Cancer. 2012;130:2857-66.

\section{Publisher's Note}

Springer Nature remains neutral with regard to jurisdictional claims in published maps and institutional affiliations. 